\title{
Investigations into the volume plasma density grating waveplate
}

\author{
George K. Holt ${ }^{\mathrm{a}}$, Gregory Vieuxa ${ }^{\mathrm{a}}$, Bernhard Ersfeld ${ }^{\mathrm{a}}$, Samuel R. Yoffe ${ }^{\mathrm{a}}$, James Feehan ${ }^{\mathrm{a}}$, Enrico \\ Brunetti $^{\mathrm{a}}$, Min Sup Hur ${ }^{\mathrm{b}}$, and Dino A. Jaroszynski ${ }^{\mathrm{a}}$

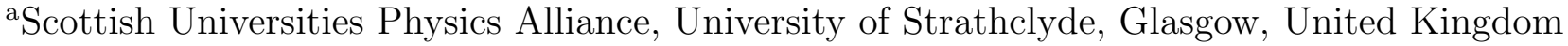 \\ ${ }^{b}$ Ulsan National Institute of Science and Technology, Ulsan, Rebuplic of Korea
}

\begin{abstract}
Volume density gratings produced by degenerate, counterpropagating laser pulses in plasma have several useful optical properties. Here we report on one of these in an investigation into creation of a transient plasma density grating that functions as a waveplate.
\end{abstract}

Keywords: plasma optics, high-intensity laser-plasma interactions, particle-in-cell simulation, plasma gratings, waveplate, polarisation of light

\section{INTRODUCTION}

Ultrashort pulse high-power lasers have advanced the understanding of fundamental physics, contributed to many applications of industrial and societal importance, and enabled numerous technological advances. Nextgeneration facilities, such as the Extreme Light Infrastructure (ELI) ${ }^{1-4}$ are based on 10s PW lasers that will generate high-brightness gamma beams, accelerate electrons to many tens of $\mathrm{GeV}$ and provide tools for investigating processes on attosecond timescales, studying nuclear physics, imaging dense matter and supporting research into new cancer therapy modalities etc.

Current high-power lasers are based on solid state optical media that have damage thresholds on the order of $1 \mathrm{~J} \mathrm{~cm}^{-2}$, for ultrashort ( $\sim 100 \mathrm{fs}$ or shorter) pulses. ${ }^{5}$ This results in large and expensive optical components; their maintenance requires careful monitoring, and damage, when it occurs, can lead to costly delays and disruption to facility access.

Plasma presents an attractive alternative optical medium for high power lasers. It is optically active, replenishable and already ionised, which results in a significantly higher damage threshold than for solid state media. Several plasma-based optical device schemes are under investigation. Plasma waveguides have been developed to guide intense laser pulse over many Rayleigh lengths, which have been used in laser wakefield accelerators to produce multi-GeV electron beams from centimetre-scale devices. ${ }^{6}$ Raman ${ }^{7,8}$ and Brillouin ${ }^{9,10}$ plasma based amplifiers are being investigated as high-gain modules for chirped pulse amplification. ${ }^{11}$ Plasma mirrors ${ }^{12}$ are routinely used to increase laser pulse contrast and generate high harmonic radiation. ${ }^{13}$

Plasma has also been proposed as a medium in which to create volume diffraction gratings, ${ }^{14,15}$ which are formed using degenerate counterpropagating laser pulses of moderate intensity $\left(\sim 1 \times 10^{15} \mathrm{~W} \mathrm{~cm}^{-2}\right)$ in subcritical density plasma, $n<n_{c}=\epsilon_{0} m_{e} \omega_{l}^{2} / e^{2}$, where $\omega_{l}$ is the angular frequency of the laser beams, and all other symbols have their usual meaning. The ponderomotive force associated with the beat wave drives electrons away from high intensity regions, while leaving higher mass ions behind. Ions gain momentum from the space-charge fields of electrons, and bunch inertially; ions and electrons form a volume plasma density grating with a periodicity equal to the beat wavelength of the "pump" laser pulses i.e. half the pump wavelength.

Figure 1 shows the temporal evolution of an ion density evolving into a volume plasma density grating, simulated using the 1-dimensional version of the particle-in-cell code EPOCH. ${ }^{16}$ The electron density distribution

Further author information: (Send correspondence to D.A.J.)

D.A.J.: E-mail: d.a.jaroszynski@strath.ac.uk

G.K.H.: E-mail: george.holt@strath.ac.uk 
is nearly identical to that of the ions. The grating is formed in plasma with an initial density $n_{0}=5 \times 10^{19} \mathrm{~cm}^{-3}$ and initial electron and ion temperatures of $T_{e}=0.5 \mathrm{eV}$ and $T_{i}=0.1 T_{e}$, respectively. The plasma grating is driven by pump pulses with wavelength $\lambda_{l}=800 \mathrm{~nm}, a_{0}=e E_{0} /\left(m_{e} \omega_{l} c\right) \approx 0.022$ (for peak laser electric field $E_{0}$ ) and Gaussian temporal envelopes with full-width half-maximum of intensity, $\tau=140 \mathrm{fs}$. The simulation uses a cell size of $0.4 \mathrm{~nm}$ and 512 macroparticles per cell.

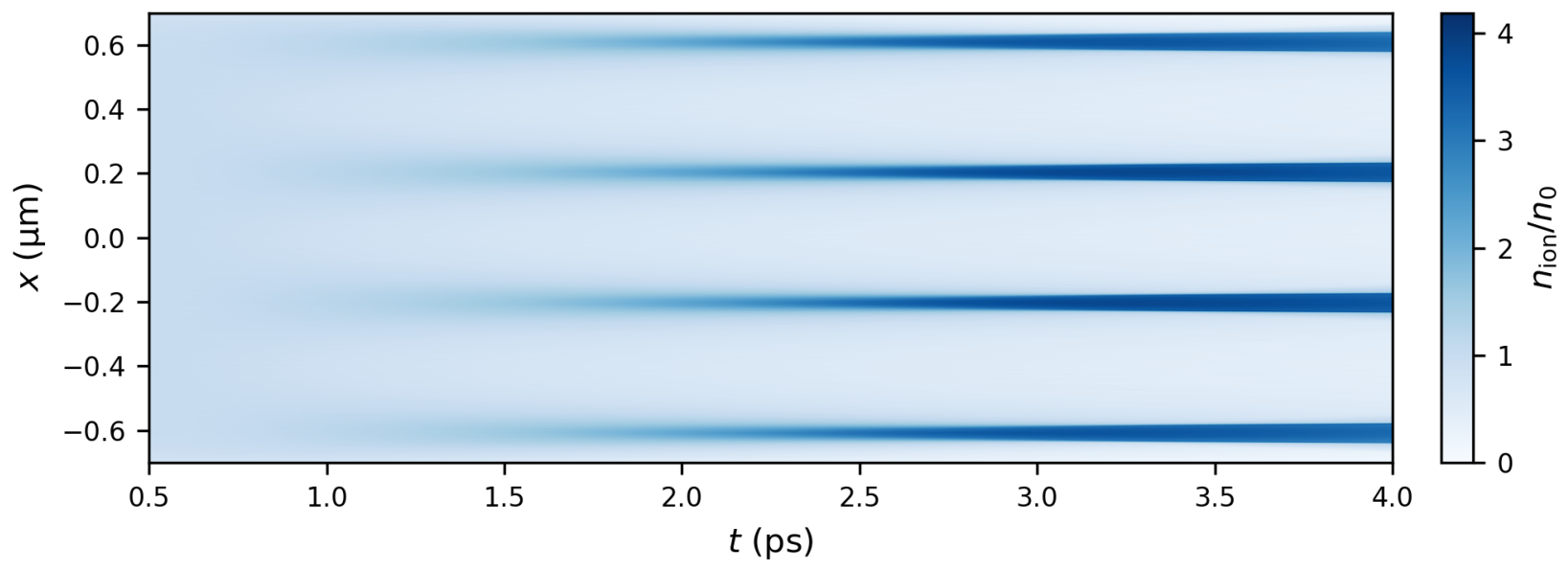

Figure 1. Evolution of a volume plasma density grating for parameters described in the text.

The timescale for creating the ion grating is estimated $\operatorname{as}^{14} T \approx\left(m_{i} / m_{e}\right)^{1 / 2}\left(k_{l}^{2} c^{2} a_{0}^{2}\right)^{-1 / 2} \approx 1 \mathrm{ps}$, where $m_{i} / m_{e}(=1836)$ is the ratio of the ion to electron mass. In our simulations we observe a grating formation time of $T \approx 2.5 \mathrm{ps}$. A fully formed density grating has a peak density nearly four times the initial value, as shown in figure 2, where ion synchrotron oscillations are evident in the phase-space plot. ${ }^{17}$

\section{VOLUME PLASMA DENSITY GRATING WAVEPLATE}

The plasma and pump pulse parameters used to generate the plasma grating should be achievable in experiments. To determine the optical properties of such a plasma grating, we employ the methods described in Ref. 18. The plasma modulation observed in the PIC simulations can be approximated as a rectangular wave with a duty cycle $a /(a+b)$ and amplitude $n_{a}-n_{b}$ (see Figure 3a). The dispersion relations for s- (TE) and p-polarised (TM) waves in the structure are determined. The dispersion relations for a wave with $\vec{k}=k \hat{y}$ (using the same geometry as in Ref. 18) are shown in Figure 3b. The slight differences in the dispersion for TE and TM waves results in birefringence. A probe laser pulse with $k_{x}=0$ will experience a relative phase difference, $\Gamma$, between the two components. This can be evaluated as a function of distance through the structure, $L$, as shown in Figure $3 c$.

\section{GRATINGS FORMED BY GAUSSIAN PUMP PULSES}

The plasma gratings considered above are produced by plane-wave pump pulses, and are therefore 1-dimensional. However, in practice, the far field spatial profiles of high-power lasers are peaked and usually assumed to be Gaussian. As the dynamics of a volume plasma density grating depends strongly on the local intensity of the pump pulses, the grating will also have a radial dependence at any given time, when created by intersecting Gaussian pump laser beams.

We have performed 2-dimensional PIC simulations using EPOCH to investigate these transverse effects, which have a bearing on the phase shift experienced by a probing laser pulse. An initially cold plasma slab with $n_{0}=6 \times 10^{19} \mathrm{~cm}^{-3}$ is considered to extend in the $x$ - and $y$-directions to a distance sufficient to encompass both the probe and radial pump fields. The pump and probe laser parameters are summarised in Table 1 . The probe is centred on $x=0$, and the pumps on $y=0$. A cell size of $\lambda_{l} / 60$ is used in both directions with 16 macroparticles per cell. 

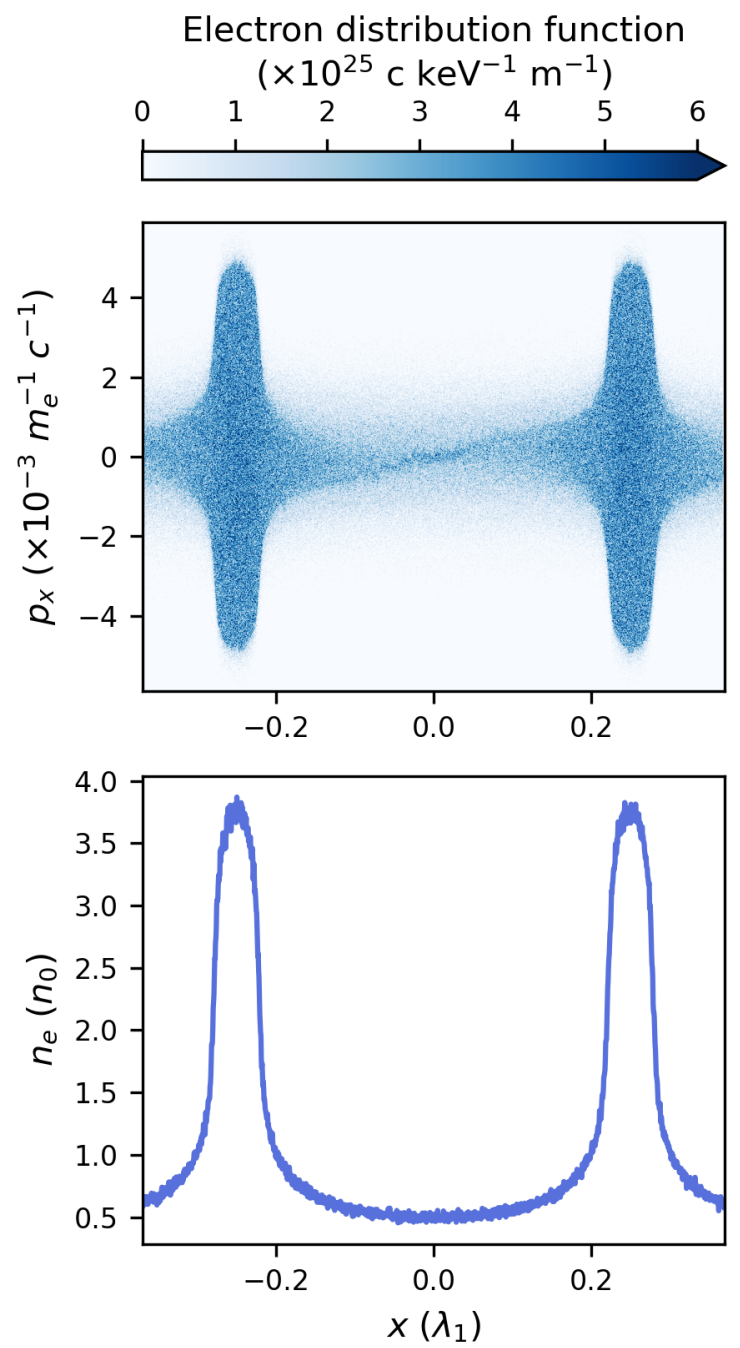
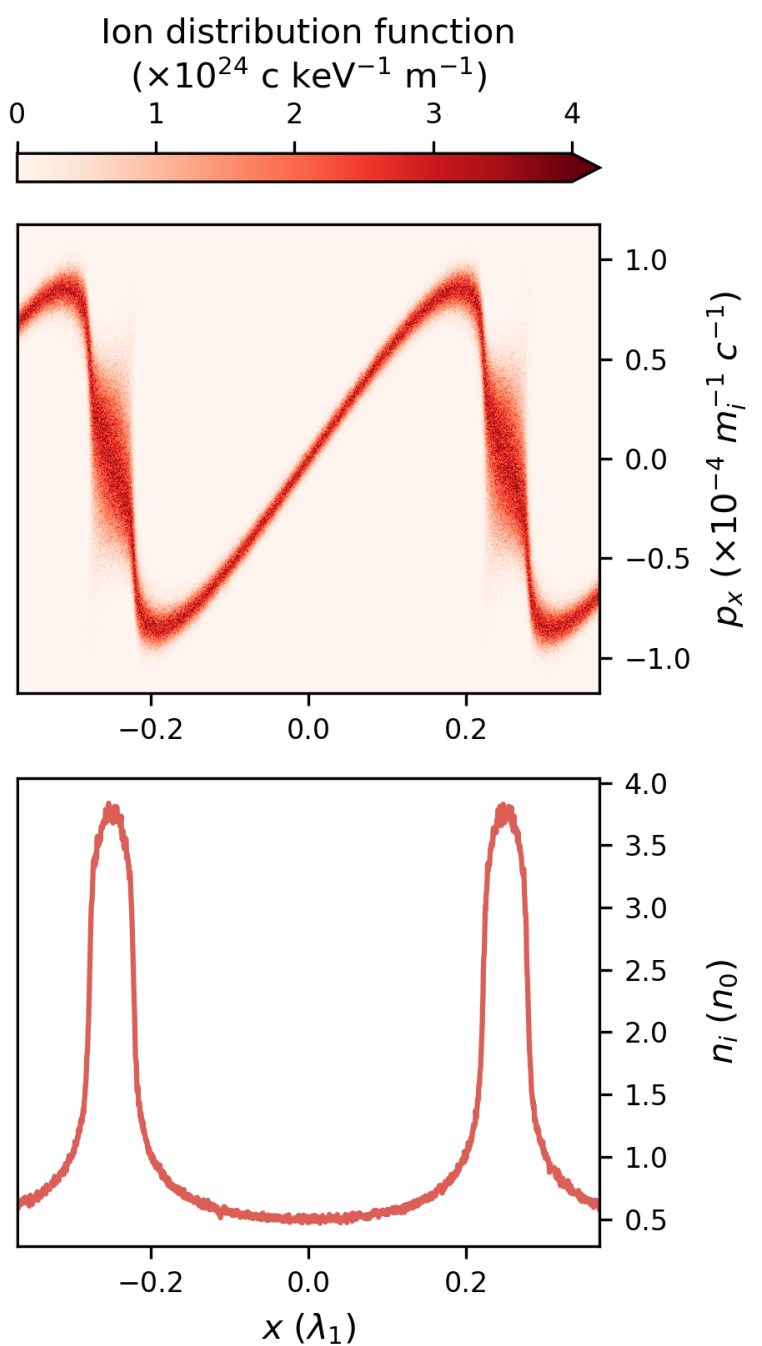

Figure 2. Phase-space distribution (top) and density (bottom) of the electrons (left, blue) and ions (right, red) in a volume plasma density grating at saturation.

A series of simulations have been performed varying the arrival time of the probe at the centre of the plasma slab, to investigate the evolution of the structure. The probe passes into vacuum where the fields can be unambiguously determined after interacting with the plasma structure. Figure 4 shows the result of a Fourier analysis of the fields to determine the relative phase changes (between the ordinary and extraordinary waves) due to the plasma grating, and how $\Gamma$ depends on the probe delay. With early delays, the probe experiences little relative phase shift because the pump fields are still ramping up and the grating is not fully formed. After $0.7 \mathrm{ps}$ the phase shift increases rapidly as birefringence grows. A peak phase shift of $21^{\circ}(\pi / 8.6)$ occurs after $0.075 \mathrm{ps}$, when the on-axis grating formation is complete, after which the structure fades and the phase shift drops gradually. The rate of decrease in phase shift is not as rapid as the build-up because the pump intensity spatial profile delays the formation times in its wings.

The time at which the peak electron density in the grating reaches a maximum coincides with the time where the largest phase shift occurs, as shown in Figure 5. Two regions of interest have been selected to highlight the radial dependence of the grating - one around $y=0$, where the pump pulse intensity is maximal (Figure 5b), and a second region further from the axis (Figure 5c). Lineouts of the plasma density in these regions are shown 

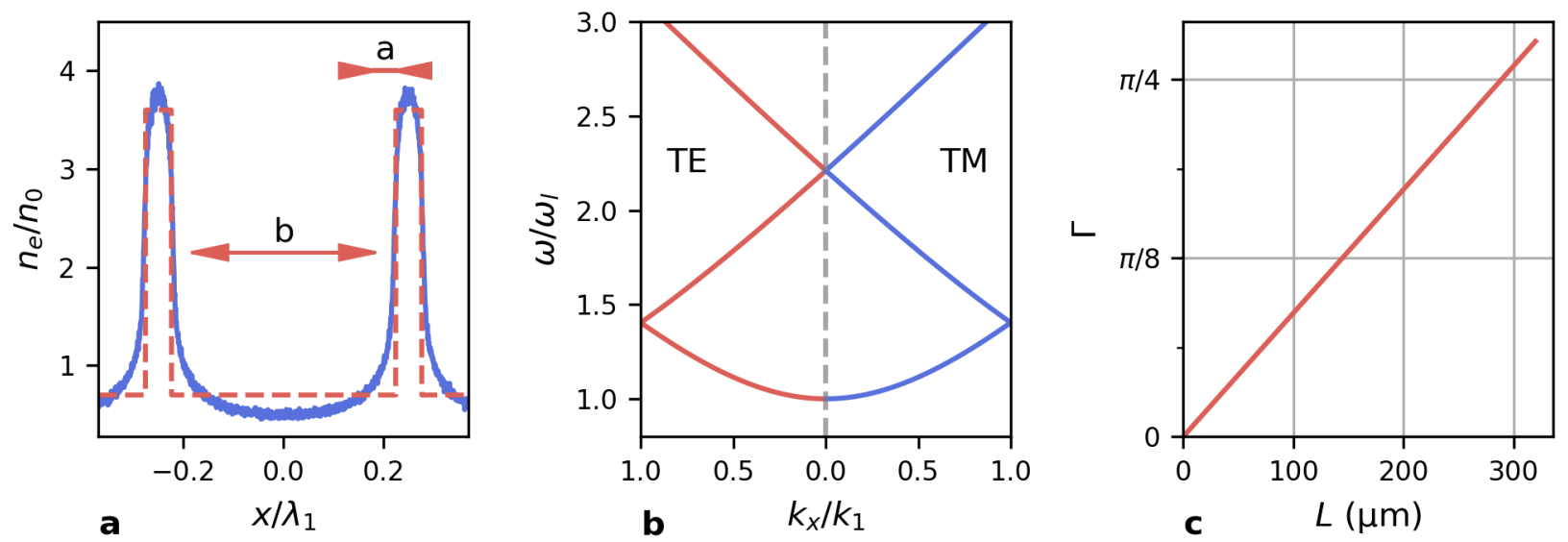

Figure 3. a Electron density of the grating at its peak value (blue solid) and the periodic density model used to calculate the dispersion relations (red dashed). b Dispersion relations for a TE (left, red) and TM (right, blue) wave with $\vec{k}=k \hat{y}$ interacting with the model plasma density grating. Wavenumber is normalised by the laser wavenumber in the unperturbed plasma, $k_{1}$. c Relative phase between TE and TM waves as they traverse the structure.

Table 1. Pump and probe parameters for the 2-dimensional PIC simulations.

\begin{tabular}{|l|l|l|}
\hline Parameter & Pumps & Probe \\
\hline$a_{0}$ & 0.0484 & $2.16 \times 10^{-3}$ \\
\hline Intensity FWHM, $\tau$ & $1 \mathrm{ps}$ & $100 \mathrm{fs}$ \\
\hline Wavelength, $\lambda$ & $800 \mathrm{~nm}$ & $800 \mathrm{~nm}$ \\
\hline Field waist, $w$ & $16 \mu \mathrm{m}$ & $6.6 \mu \mathrm{m}$ \\
\hline Propagation direction & $\hat{x}$ & $\hat{y}$ \\
\hline
\end{tabular}

in Figs. 5d,e, indicated by the dashed and dot-dashed lines. At this time, the grating has peaked and is decaying at $y=0$, and formation is peaked at $|y| \approx 6 \mu \mathrm{m}$. The relative phase differences between TE and TM components is integrated across the grating.

\section{CONCLUSIONS}

Plasma optics could become important elements in next-generation high-power lasers as they push beyond $10 \mathrm{PW}$ powers. Here we have investigated how counterpropagating Gaussian laser pulses can be used to create a volume plasma density grating for use as a waveplate. These considerations are necessary for planning experimental investigations into the future use of volume plasma density gratings.

\section{ACKNOWLEDGMENTS}

This work was supported by the U.K. EPSRC (grant number EP/N028694/1) and received funding from the European Union's Horizon 2020 research and innovation programme Laserlab Europe (grant number 871124). This work used the ARCHER UK National Supercomputing Service (http://www.archer.ac.uk), the ARCHER2 UK National Supercomputing Service (https://www.archer2.ac.uk) and the Cirrus UK National Tier-2 HPC Service at EPCC (http://www.cirrus.ac.uk) funded by the University of Edinburgh and EPSRC (EP/P020267/1). Development of the EPOCH code is supported by EPSRC (EP/G054950/1, EP/G056803/1, EP/G055165/1, $\mathrm{EP} / \mathrm{M} 022463 / 1)$. Data associated with research published in this paper is available at https://doi.org/10.15129/c00199b7-a63b-41b3-bcc6-55524b8699c3. 


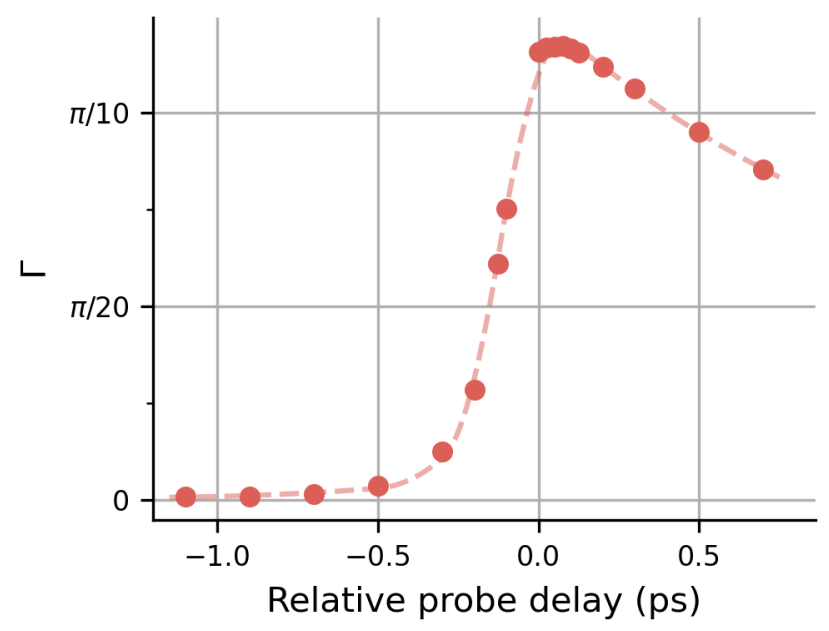

Figure 4. 2-dimensional simulations showing the dependence of the probe phase shift on arrival time relative to the pump pulses.

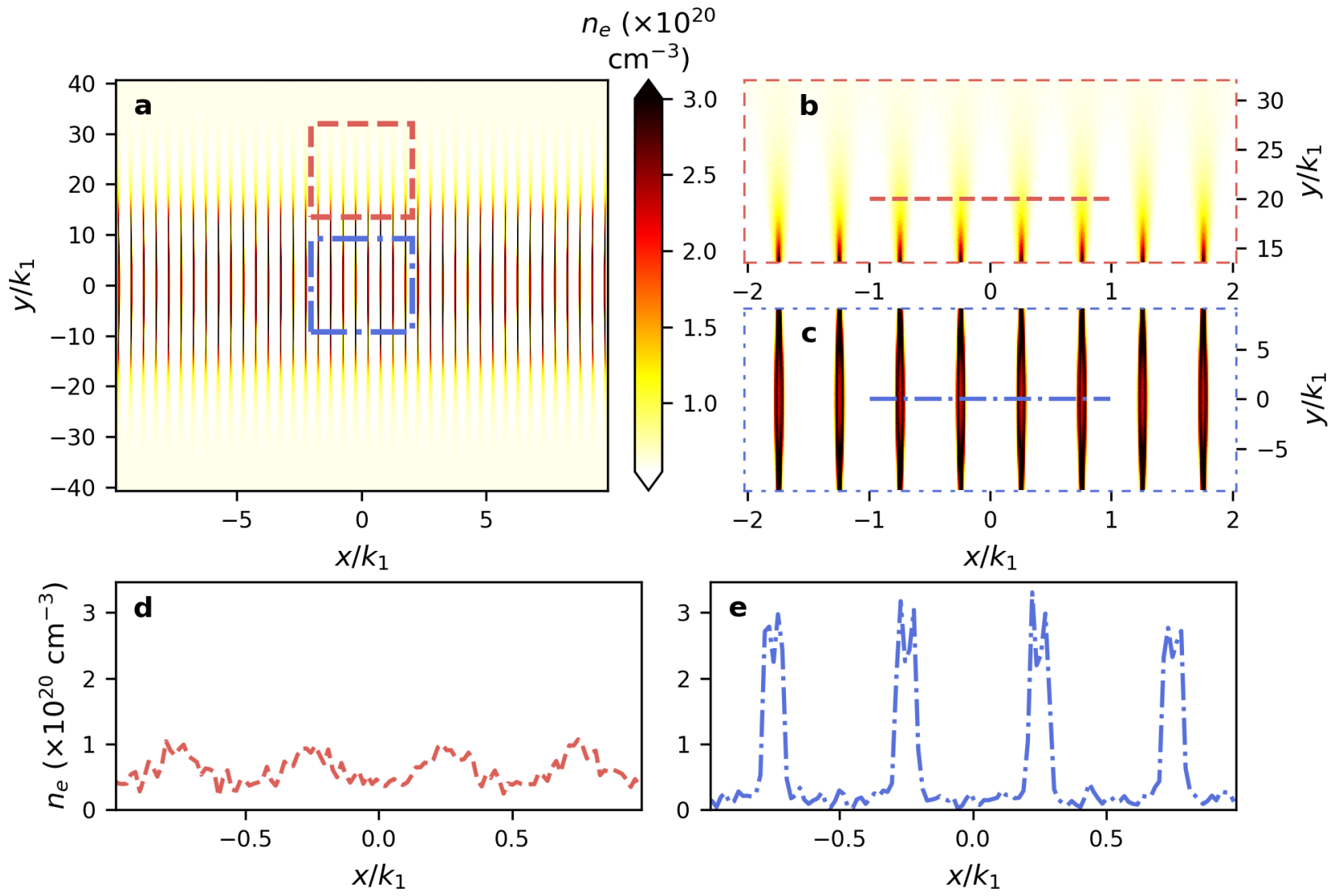

Figure 5. Electron density of a plasma grating formed by Gaussian pump pulses. The regions of interested marked by the red dashed and blue dot-dashed boxes in $\mathbf{a}$ are shown in $\mathbf{b}$ and $\mathbf{c}$, respectively. The upper and lower limits of the colour scale have been truncated to emphasise elements of the grating. The lineouts indicated in $\mathbf{b}$ and $\mathbf{c}$ are shown in $\mathbf{d}$ and $\mathbf{e}$, respectively. 


\section{REFERENCES}

[1] Gérard, M. A., Korn, G., Sandner, W., and Collier, J. L., "ELI - Extreme Light Infrastructure Science and Technology with Ultra-Intense Lasers," (2011).

[2] Rus, B., Bakule, P., Kramer, D., Naylon, J., Thoma, J., Fibrich, M., Green, J. T., Lagron, J. C., Antipenkov, R., Bartoníček, J., Batysta, F., Baše, R., Boge, R., Buck, S., Cupal, J., Drouin, M. A., Durák, M., Himmel, B., Havlíček, T., Homer, P., Honsa, A., Horáček, M., Hríbek, P., Hubáček, J., Hubka, Z., Kalinchenko, G., Kasl, K., Indra, L., Korous, P., Košelja, M., Koubíková, L., Laub, M., Mazanec, T., Meadows, A., Novák, J., Peceli, D., Polan, J., Snopek, D., Šobr, V., Trojek, P., Tykalewicz, B., Velpula, P., Verhagen, E., Vyhlídka, Š., Weiss, J., Haefner, C., Bayramian, A., Betts, S., Erlandson, A., Jarboe, J., Johnson, G., Horner, J., Kim, D., Koh, E., Marshall, C., Mason, D., Sistrunk, E., Smith, D., Spinka, T., Stanley, J., Stolz, C., Suratwala, T., Telford, S., Ditmire, T., Gaul, E., Donovan, M., Frederickson, C., Friedman, G., Hammond, D., Hidinger, D., Chériaux, G., Jochmann, A., Kepler, M., Malato, C., Martinez, M., Metzger, T., Schultze, M., Mason, P., Ertel, K., Lintern, A., Edwards, C., Hernandez-Gomez, C., and Collier, J., "ELI-beamlines: Progress in development of next generation short-pulse laser systems," in [Research Using Extreme Light: Entering New Frontiers with Petawatt-Class Lasers III], 10241, 102410J, International Society for Optics and Photonics (June 2017).

[3] Gales, S., Tanaka, K. A., Balabanski, D. L., Negoita, F., Stutman, D., Tesileanu, O., Ur, C. A., Ursescu, D., Andrei, I., Ataman, S., Cernaianu, M. O., D’Alessi, L., Dancus, I., Diaconescu, B., Djourelov, N., Filipescu, D., Ghenuche, P., Ghita, D. G., Matei, C., Seto, K., Zeng, M., and Zamfir, N. V., "The extreme light infrastructure - nuclear physics (ELI-NP) facility: New horizons in physics with $10 \mathrm{PW}$ ultra-intense lasers and $20 \mathrm{MeV}$ brilliant gamma beams," Reports Prog. Phys. 81, 094301 (Sept. 2018).

[4] Kühn, S., Dumergue, M., Kahaly, S., Mondal, S., Füle, M., Csizmadia, T., Farkas, B., Major, B., Várallyay, Z., Cormier, E., Kalashnikov, M., Calegari, F., Devetta, M., Frassetto, F., M $\backslash$ aansson, E., Poletto, L., Stagira, S., Vozzi, C., Nisoli, M., Rudawski, P., Maclot, S., Campi, F., Wikmark, H., Arnold, C. L., Heyl, C. M., Johnsson, P., L'Huillier, A., Lopez-Martens, R., Haessler, S., Bocoum, M., Boehle, F., Vernier, A., Iaquaniello, G., Skantzakis, E., Papadakis, N., Kalpouzos, C., Tzallas, P., Lépine, F., Charalambidis, D., Varjú, K., Osvay, K., and Sansone, G., "The ELI-ALPS facility: The next generation of attosecond sources," Journal of Physics B: Atomic, Molecular and Optical Physics 50, 132002 (June 2017).

[5] Tien, A.-C., Backus, S., Kapteyn, H., Murnane, M., and Mourou, G., "Short-Pulse Laser Damage in Transparent Materials as a Function of Pulse Duration," Physical Review Letters 82, 3883-3886 (May 1999).

[6] Gonsalves, A. J., Nakamura, K., Daniels, J., Benedetti, C., Pieronek, C., De Raadt, T. C., Steinke, S., Bin, J. H., Bulanov, S. S., Van Tilborg, J., Geddes, C. G., Schroeder, C. B., Tóth, C., Esarey, E., Swanson, K., Fan-Chiang, L., Bagdasarov, G., Bobrova, N., Gasilov, V., Korn, G., Sasorov, P., and Leemans, W. P., "Petawatt Laser Guiding and Electron Beam Acceleration to $8 \mathrm{GeV}$ in a Laser-Heated Capillary Discharge Waveguide," Phys. Rev. Lett. 122 (Feb. 2019).

[7] Malkin, V. M., Shvets, G., and Fisch, N. J., "Fast Compression of Laser Beams to Highly Overcritical Powers," Physical Review Letters 82, 4448-4451 (May 1999).

[8] Vieux, G., Cipiccia, S., Grant, D. W., Lemos, N., Grant, P., Ciocarlan, C., Ersfeld, B., Hur, M. S., Lepipas, P., Manahan, G. G., Raj, G., Reboredo Gil, D., Subiel, A., Welsh, G. H., Wiggins, S. M., Yoffe, S. R., Farmer, J. P., Aniculaesei, C., Brunetti, E., Yang, X., Heathcote, R., Nersisyan, G., Lewis, C. L. S., Pukhov, A., Dias, J. M., and Jaroszynski, D. A., "An ultra-high gain and efficient amplifier based on Raman amplification in plasma," Scientific Reports 7, 2399 (May 2017).

[9] Milroy, R. D., Capjack, C. E., and James, C. R., "Plasma laser pulse amplifier using induced Raman or Brillouin processes," The Physics of Fluids 22, 1922-1931 (Oct. 1979).

[10] Marquès, J.-R., Lancia, L., Gangolf, T., Blecher, M., Bolaños, S., Fuchs, J., Willi, O., Amiranoff, F., Berger, R. L., Chiaramello, M., Weber, S., and Riconda, C., "Joule-Level High-Efficiency Energy Transfer to Subpicosecond Laser Pulses by a Plasma-Based Amplifier," Physical Review X 9, 021008 (Apr. 2019).

[11] Ersfeld, B. and Jaroszynski, D. A., "Superradiant Linear Raman Amplification in Plasma Using a Chirped Pump Pulse," Physical Review Letters 95, 165002 (Oct. 2005). 
[12] Kapteyn, H. C., Murnane, M. M., Szoke, A., and Falcone, R. W., "Prepulse energy suppression for highenergy ultrashort pulses using self-induced plasma shuttering," Optics Letters 16, 490-492 (Apr. 1991).

[13] Wheeler, J. A., Borot, A., Monchocé, S., Vincenti, H., Ricci, A., Malvache, A., Lopez-Martens, R., and Quéré, F., "Attosecond lighthouses from plasma mirrors," Nature Photonics 6, 829-833 (Dec. 2012).

[14] Sheng, Z.-M., Zhang, J., and Umstadter, D., "Plasma density gratings induced by intersecting laser pulses in underdense plasmas," Appl. Phys. B 77, 673-680 (Nov. 2003).

[15] Lehmann, G. and Spatschek, K. H., "Transient Plasma Photonic Crystals for High-Power Lasers," Phys. Rev. Lett. 116, 225002 (June 2016).

[16] Arber, T. D., Bennett, K., Brady, C. S., Lawrence-Douglas, A., Ramsay, M. G., Sircombe, N. J., Gillies, P., Evans, R. G., Schmitz, H., Bell, A. R., and Ridgers, C. P., "Contemporary particle-in-cell approach to laser-plasma modelling," Plasma Phys. Control. Fusion 57, 113001 (Nov. 2015).

[17] Peng, H., Riconda, C., Grech, M., Su, J.-Q., and Weber, S., "Nonlinear dynamics of laser-generated ionplasma gratings: A unified description," Physical Review E 100, 061201 (Dec. 2019).

[18] Lehmann, G. and Spatschek, K. H., "Plasma-based polarizer and waveplate at large laser intensity," Phys. Rev. E 97, 063201 (June 2018). 